\title{
Performance Evaluation of Phase Estimator Using LMS, Variable Step Size LMS and RLS Algorithm for QAM Communication Systems
}

\author{
${ }^{1}$ Ankit Aggarwal, ${ }^{2}$ Baljit Kaur, ${ }^{3}$ Kamlesh Yadav \\ ${ }^{I}$ M.Tech Student, ECE Deptt. SSIET, Derabassi, Punjab, India \\ ${ }^{2}$ Asst. Prof., ECE Deptt., SSIET, Derabassi, Punjab, India \\ ${ }^{3}$ Asst Prof., ECE Deptt., M.M.U., Mullana, Ambala, Haryana, India
}

\begin{abstract}
Phase estimation is a problem of paramount importance in synchronous digital communication system, especially for high bit rate signaling such as QAM modulation. QAM is particularly attractive for highthroughput-efficiency application and better performance compared to PSK as size of constellation increases. Ideally, the phase estimation must be performed in a blind manner, means without using known training sequences of known transmitted symbols. To better understand, consider data is transmitted by transmitter using 8-QAM modulation. The data will be divided into symbol that consist of 3 bits per symbol and each modulated data will be placed at specific angle $\left(-45^{\circ},+45^{\circ},-135^{\circ},+135^{\circ}\right.$ in this case) [5] and transmitted through the common channel. At the receiver, it is required to know this phase angle accurately; else the demodulated data will encounter errors. Therefore, the accuracy in phase recovery is very important.

Automatic equalizers use iterative techniques to estimate the optimum coefficients. To obtained a stable solution to the filter weights, it is necessary that the data be averaged to obtain stable signal statistics, or the noisy solution obtained from the noisy data must be averaged. The most robust of this class of algorithm is the Variable step size least mean square (VSSLMS) algorithm. Each iteration of this algorithm uses a noisy estimate of the error gradient to adjust the weights in the direction to reduce the average mean square error. Noisy gradient is simply the product of data vector and error scalar.

The data vector is the vector of noise-corrupted channel samples residing in the equalizer filter at a given time. This paper focuses on LMS; Variable step size LMS and RLS (Recursive Least Square) based adaptive algorithms.
\end{abstract}

Keywords: $L M S, R L S$, VSSLMS, Adaptive Filters

\section{Introduction to Adaptive Filters:}

Phase offset simply means change in phase of received signal from the transmitted signal.

Potential source of phase offset are:

- Transmission delay

- Environmental noise

- The phase instability in oscillators

The Adaptive filters are the solution for phase estimation and also in practical situations, the system is operating in an uncertain environment where the input condition is not clear and/or the unexpected noise exists. Under such circumstances, the system should have the flexible ability to modify the system parameters and makes the adjustments based on input signal and the other relevant signal to obtain an optimal performance.

A system that searches for improved performance guided by a computational algorithm for adjustment of the parameters or weights is called an adaptive system. The adaptive system is time varying.

During the last decades, the adaptive filters have attracted the attention of many researchers due to their property of self-designing. In applications where some a priori information about the statistics of the data is available, a linear filter optimal for those applications can be designed in advance. In the absence of this priori information, a solution is to use adaptive filters which possess the ability to adapt their coefficients as a function of the filtering error. As a consequence, the adaptive filters and algorithms were successfully implemented in a wide variety of devices for diverse applications fields such as communication, control, radar and biomedical engineering etc. The basic filter structure is as shown in figure 1.1. 


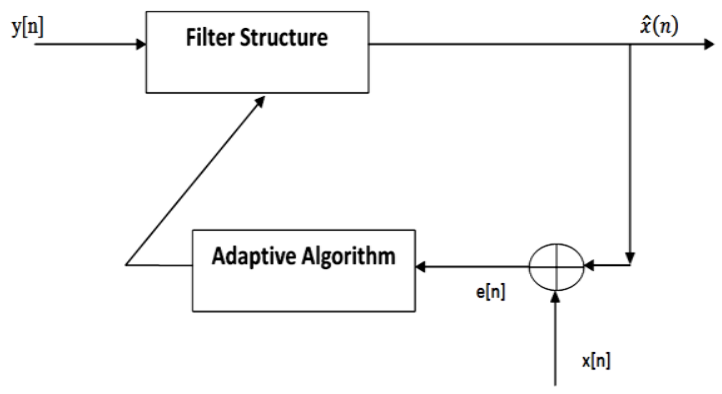

Figure 1.1: Block Diagram of updating weights of Filter using Adaptive algorithm [1]

\section{Adaptation Algorithms:}

There are many different types of adaptive algorithms that can be used with an equalizer. These algorithms can adapt themselves based on any number of criteria.

The two adaptation algorithms taken into consideration in this Chapter are Least Mean Square and Recursive Least Square filter. LMS is most widely used adaptive algorithms because of its simplicity, but has a relatively slow convergence rate. The RLS algorithms, on the other hand, are more complex than the LMS algorithm, but provide a much faster rate of convergence. Both of these algorithms are discussed below.

\section{Least Mean Squares Algorithm:}

Least mean squares algorithm is a class of adaptive filter used to design the desired filter by finding the filter coefficients that relate to producing the least mean squares of the error signal (difference between the desired and actual signal). It is a stochastic gradient method in which the filter is only adapted based on the error at the current time. LMS filter is built around a transversal (i.e. tapped delay line) structure [2]. LMS filter employ, small step size statistical theory, which provides a fairly accurate description of the transient behaviour.

The LMS algorithm in general, consists of two basics procedure. The first step is filtering process, which involve, computing the output of a linear filter in response to the input signal, and the second step is generating an estimation error by comparing this output with a desired response as follows:

$$
\mathrm{e}[\mathrm{n}]=\mathrm{x}[\mathrm{n}]-\hat{\mathrm{x}}(\mathrm{n})
$$

Where, $y(n)$ is filter output and $d(n)$ is the desired response at time $n$. Adaptive process, which involves the automatic adjustment of the parameter of the filter in accordance with the estimation error, is given by:

$$
\begin{aligned}
& \mathrm{h}[\mathrm{n}+1]=\mathrm{h}[\mathrm{n}]+\frac{\mu}{2}\left(-\nabla \mathrm{Ee}^{2}[\mathrm{n}]\right) \\
& \text { Where, } \nabla \mathrm{Ee}^{2}[\mathrm{n}]=\left[\begin{array}{c}
\frac{\partial \mathrm{Ee}^{2}[\mathrm{n}]}{\partial \mathrm{h}_{0}} \\
\cdots \cdots . \\
\cdots \ldots . \\
\ldots \ldots . \\
\frac{\partial \mathrm{Ee}^{2}[\mathrm{n}]}{\partial \mathrm{h}_{\mathrm{M}-1}}
\end{array}\right]
\end{aligned}
$$

$\mu$ is the step size, $(n+1)=$ estimate of tape weight vector at time $(n+1)$ and if prior knowledge of the tape weight vector ( $\mathrm{n}$ ) is not available, then $\mathrm{n}$ is set to 0 . The combination of these two processes working together constitutes a feedback loop. Firstly using a transversal filter, the LMS algorithm is built, and the same is responsible for performing the filtering process. Secondly, the adaptive control process on the tap weight of the transversal filter is performed.

In the LMS algorithm, $\mathrm{Ee}^{2}[\mathrm{n}]$ is approximated by $\mathrm{e}[\mathrm{n}]$ to achieve a computationally simple algorithm. 
$\nabla \mathrm{Ee}^{2}[\mathrm{n}] \approx 2 . \mathrm{e}[\mathrm{n}] .\left[\begin{array}{l}\frac{\partial \mathrm{e}[\mathrm{n}]}{\partial \mathrm{h}_{0}} \\ \cdots \cdots \\ \cdots \cdots \\ \cdots \cdots . . \\ \frac{\partial \mathrm{e}[\mathrm{n}]}{\partial \mathrm{h}_{\mathrm{M}}-1}\end{array}\right]$

Now consider,

$e[n]=x[n]-\sum_{i=0}^{M-1} h_{i}[n] y[n-i]$
$\frac{\partial e[n]}{\partial h_{j}}=-y[n-j], j=0,1, \ldots \ldots \ldots M-1$

Therefore,

$\left[\begin{array}{c}\frac{\partial e[n]}{\partial \mathrm{h}_{0}} \\ \cdots \cdots \cdots \\ \cdots \cdots \\ \cdots \ldots . . \\ \frac{\partial \mathrm{e}[\mathrm{n}]}{\partial \mathrm{h}_{\mathrm{M}-1}}\end{array}\right]=-\left[\begin{array}{c}\mathrm{y}[\mathrm{n}] \\ \mathrm{y}[\mathrm{n}-1] \\ \cdot \\ \cdot \\ \cdot \\ y[\mathrm{n}-\mathrm{M}+1]\end{array}\right]=-\mathrm{y}[\mathrm{n}]$

Therefore, $\nabla \mathrm{Ee}^{2}[\mathrm{n}]=-2 \mathrm{e}[\mathrm{n}] \mathrm{y}[\mathrm{n}]$

The steepest descent update now becomes

$\mathrm{h}[\mathrm{n}+1]=\mathrm{h}[\mathrm{n}]+\mu \mathrm{e}[\mathrm{n}] \mathrm{y}[\mathrm{n}]$

This modification is due to Windrow and Hopf and the corresponding adaptive filter is known as the LMS filter.

Hence the LMS algorithm is as follows:

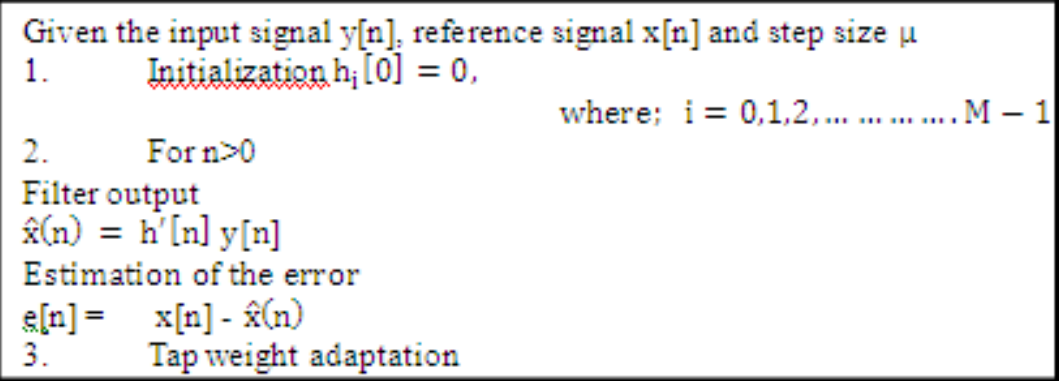

$\mathrm{h}[\mathrm{n}+1]=\mathrm{h}[\mathrm{n}]+\mu \mathrm{e}[\mathrm{n}] \mathrm{y}[\mathrm{n}]$

\section{Convergence of LMS algorithm}

As there is a feedback in the adaptive algorithm, convergence is generally not assured. The convergence of the algorithm depends on the step size parameter $\mu$.

The LMS algorithm is convergent in the mean if the step size parameter $\mu$ satisfies the condition

$$
0<\mu<\frac{2}{\lambda_{\max }}
$$

Generally, a too small value of $\mu$ results in slower convergence where as big values of $\mu$ will result in larger fluctuations from the mean. Value of $\mu$ lies between 0 and 1 [3]. Choosing a proper value of $\mu$ is very important for the performance of the LMS algorithm.

\section{Recursive Least Square Algorithm:}

The Recursive least squares adaptive filter is an algorithm which recursively finds the filter coefficients that minimize a weighted linear least squares function relating to the input signals. This is in contrast to the LMS that aim to reduce the mean square error. In the derivation of the RLS, the input signals are considered deterministic, while for the LMS, they are considered stochastic. However, the RLS exhibits extremely fast convergence [4-5]. But, this benefit comes at the cost of high computational complexity, and potentially poor tracking performance when the filter to be estimated changes. The RLS algorithm has the same procedures as 
LMS algorithm, except that it provides a tracking rate sufficient for fast fading channel.

The RLS algorithm is known to have the stability issues due to the covariance update formula $p(n)$, which is used for automatic adjustment in accordance with the estimation error .

The RLS algorithm considers all the available data for determining the filter parameters. The filter should be optimum with respect to all the available data in certain sense [6].

Minimizes the cost function

$\varepsilon[\mathrm{n}]=\sum_{\mathrm{k}=0}^{\mathrm{n}} \lambda^{\mathrm{n}-\mathrm{k}} \mathrm{e}^{2}[\mathrm{k}]$

With respect to the filter parameter vector $h[n]=\left[\begin{array}{c}h_{0}[n] \\ h_{1}[n] \\ \cdot \\ \cdot \\ h_{M-1}[n]\end{array}\right]$

Where $\lambda$ is the weighing factor known as the forgetting factor..

- $\quad$ Recent data is given more weightage.

- $\quad$ For stationary case $\lambda=1$ can be taken.

- $\lambda \cong 0.99$ is effective in tracking local nonstationarity.

The minimization problem is

Minimize

$\varepsilon[\mathrm{n}]=\sum_{\mathrm{k}=0}^{\mathrm{n}} \lambda^{\mathrm{n}-\mathrm{k}}\left(\mathrm{x}[\mathrm{k}]-\mathrm{y}^{\prime}[\mathrm{k}] \mathrm{h}[\mathrm{n}]\right)^{2}$

with respect to $h[n]$

The minimum is given by

$$
\frac{\partial \varepsilon(\mathrm{n})}{\partial \mathrm{h}(\mathrm{n})}=0
$$

Let us define

$$
<=2 \sum_{\mathrm{k}=0}^{\mathrm{n}} \lambda^{\mathrm{n}-\mathrm{k}}\left(\mathrm{x}[\mathrm{k}] \mathrm{y}[\mathrm{k}]-\mathrm{y}[\mathrm{k}] \mathrm{y}^{\prime}[\mathrm{k}] \mathrm{h}[\mathrm{n}]\right)=0
$$

$$
<=\mathrm{h}[\mathrm{n}]\left(\sum_{\mathrm{k}=0}^{\mathrm{n}} \lambda^{\mathrm{n}-\mathrm{k}} \mathrm{y}[\mathrm{k}] \mathrm{y}^{\prime}[\mathrm{k}]\right)^{-1} \sum_{\mathrm{k}=0}^{\mathrm{n}} \lambda^{\mathrm{n}-\mathrm{k}} \mathrm{x}[\mathrm{k}] \mathrm{y}[\mathrm{k}]
$$

$\widehat{\mathrm{R}}_{\mathrm{yy}}[\mathrm{n}]=\sum_{\mathrm{k}=0}^{\mathrm{n}} \lambda^{\mathrm{n}-\mathrm{k}} \mathrm{y}[\mathrm{k}] \mathrm{y}^{\prime}[\mathrm{k}]$

This is an estimator for the autocorrelation matrix $R_{y y}$.

Similarly, $\widehat{r}_{\mathrm{xy}}[\mathrm{n}]=\sum_{\mathrm{k}=0}^{\mathrm{n}} \lambda^{\mathrm{n}-\mathrm{k}} \mathrm{x}[\mathrm{k}] \mathrm{y}[\mathrm{k}]=$ estimator for the autocorrelation vector $\mathrm{r}_{\mathrm{xy}}[\mathrm{n}]$.

Hence $h[n]=\left(\widehat{R}_{y y}[n]\right)^{-1} \widehat{r}_{x y}[n]$

Matrix inversion is involved which makes the direct solution difficult. We look forward for a recursive solution.

Recursive Representation of $\widehat{\mathbf{R}}_{\mathbf{y y}}[\mathbf{n}]$ :

$\widehat{\mathrm{R}}_{\mathrm{yy}}[\mathrm{n}]$ can be written as follows

$$
\begin{aligned}
& \widehat{\mathrm{R}}_{\mathrm{yy}}[\mathrm{n}]=\sum_{\mathrm{k}=0}^{\mathrm{n}-1} \lambda^{\mathrm{n}-\mathrm{k}} \mathrm{y}[\mathrm{k}] \mathrm{y}^{\prime}[\mathrm{k}]+\mathrm{y}[\mathrm{n}] \mathrm{y}^{\prime}[\mathrm{n}] \\
& =\lambda \sum_{\mathrm{k}=0}^{\mathrm{n}-1} \lambda^{\mathrm{n}-1-\mathrm{k}} \mathrm{y}[\mathrm{k}] \mathrm{y}^{\prime}[\mathrm{k}]+\mathrm{y}[\mathrm{n}] \mathrm{y}^{\prime}[\mathrm{n}] \\
& =\lambda \widehat{\mathrm{R}}_{\mathrm{yy}}[\mathrm{n}-1]+\mathrm{y}[\mathrm{n}] \mathrm{y}^{\prime}[\mathrm{n}]
\end{aligned}
$$

This shows that the autocorrelation matrix can be recursively computed from its previous values and the present data vector.

$$
\text { Similarly, } \hat{r}_{x y}[n]=\lambda \hat{r}_{x y}[n-1]+x[n] y[n]
$$




$$
\begin{aligned}
& \mathrm{h}[\mathrm{n}]=\left(\widehat{\mathrm{R}}_{\mathrm{yy}}[\mathrm{n}]\right)^{-1} \hat{\mathrm{r}}_{\mathrm{xy}}[\mathrm{n}] \\
& =\lambda\left(\widehat{\mathrm{R}}_{\mathrm{yy}}[\mathrm{n}-1]+\mathrm{y}[\mathrm{n}] \mathrm{y}^{\prime}[\mathrm{n}]\right)^{-1} \widehat{\mathrm{r}}_{\mathrm{xy}}[\mathrm{n}]
\end{aligned}
$$

For the matrix inversion above the matrix inversion lemma will be useful.

By using Matrix inversion lemma, we get

$$
P[n]=\frac{1}{\lambda}\left(P[n-1]-k[n] y^{\prime}[n] P[n-1]\right)
$$

Where $\mathrm{k}[\mathrm{n}]$ is called 'gain vector' and given by

$$
\mathrm{k}[\mathrm{n}]=\frac{\mathrm{P}[\mathrm{n}-1]) \mathrm{y}[\mathrm{n}]}{\lambda+\mathrm{y}^{\prime}[\mathrm{n}] \mathrm{P}[\mathrm{n}-1] \mathrm{y}[\mathrm{n}]}
$$

And $\mathrm{P}[\mathrm{n}]=\widehat{\mathrm{R}}_{\mathrm{yy}}{ }^{-1}[\mathrm{n}]$

$\mathrm{k}[\mathrm{n}]$ important to interpret adaptation is also related to the current data vector $\mathrm{y}[\mathrm{n}]$ by

$\mathrm{k}[\mathrm{n}]=\mathrm{P}[\mathrm{n}] \mathrm{y}[\mathrm{n}]$

To establish the above relation consider

$\mathrm{P}[\mathrm{n}]=\frac{1}{\lambda}\left(\mathrm{P}[\mathrm{n}-1]-\mathrm{k}[\mathrm{n}] \mathrm{y}^{\prime}[\mathrm{n}] \mathrm{P}[\mathrm{n}-1]\right)$

\section{RLS algorithm Steps:}

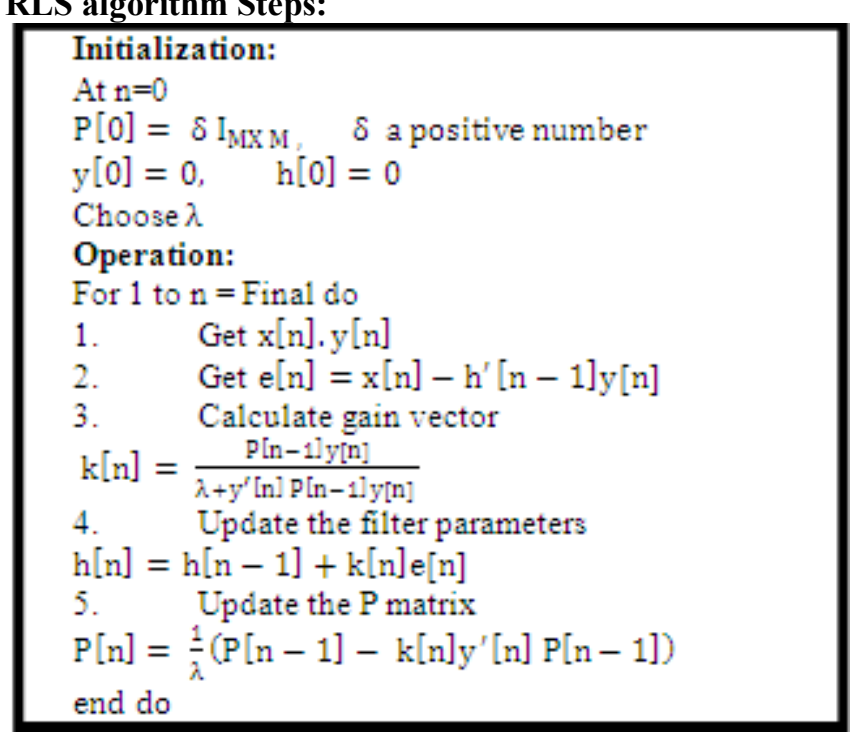

Simulation Results: The above two algorithms are implemented in Matlab to recover the phase. The model employed consists of random data generator for 16- QAM. This data is modulated using QAM modulation with constellation size 16- QAM The modulated signals are processed and then passed through Additive White Gaussian Noise (AWGN) channel.

In this model, the SNR is $26.0206 \mathrm{~dB}$. The simulation process is carried out at different - different no. of weights, at different step sizes, at different phase rotations (The phase noise is introduced artificially leading to a phase rotation of $10,20 \& 30$ degrees).

The recovered equalised signal is then demodulated using QAM demodulator and the symbol error rate (SER) is then computed between the equalised received signal and the input signal. The output is observed in the form of SER (Symbol Error Rate).

The Simulation results are shown below. 


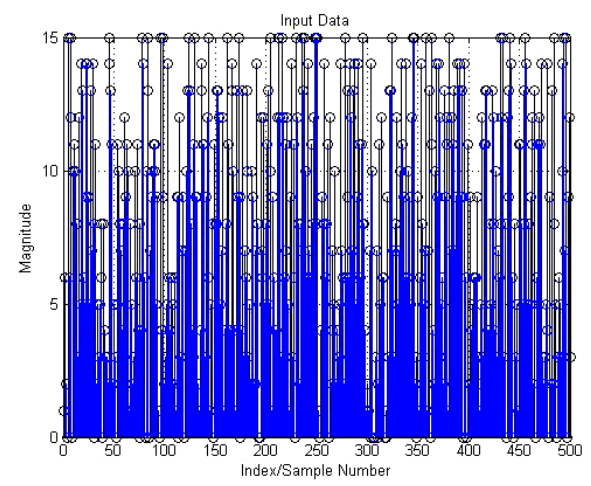

Figure 1.2: A typical example of the simulated input data.

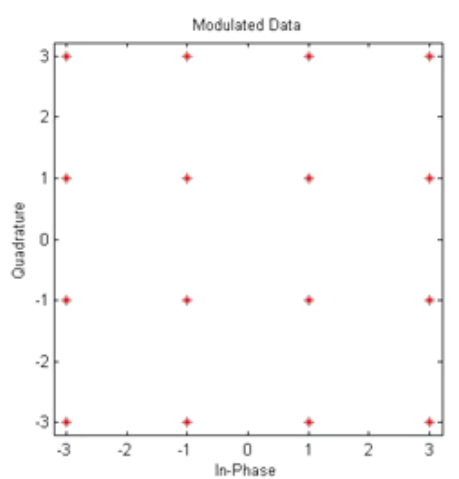

Figure 1.3: A typical example of the QAM (Constellation Size $=16)$ modulated data.

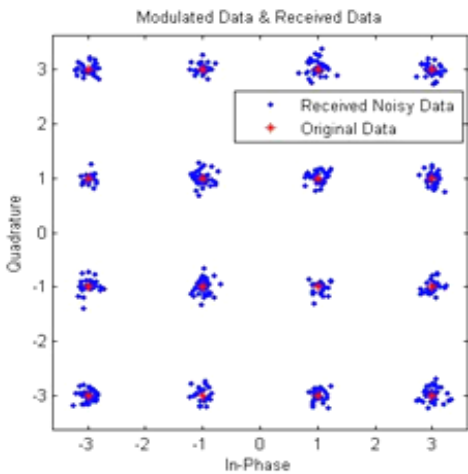

Figure 1.4: The received modulated data with noise (SNR is $26 \mathrm{~dB} \&$ EbNo is $20 \mathrm{~dB}$ ).

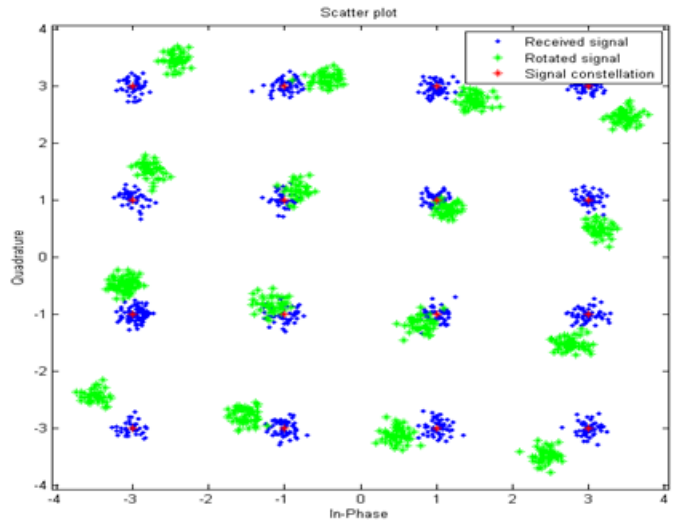

Figure 1.5: The received noisy modulated data with rotated phase of 10 degrees ( $\mathrm{SNR}$ is $26.0206 \mathrm{~dB} \&$ EbNo is $20 \mathrm{~dB}$ ).

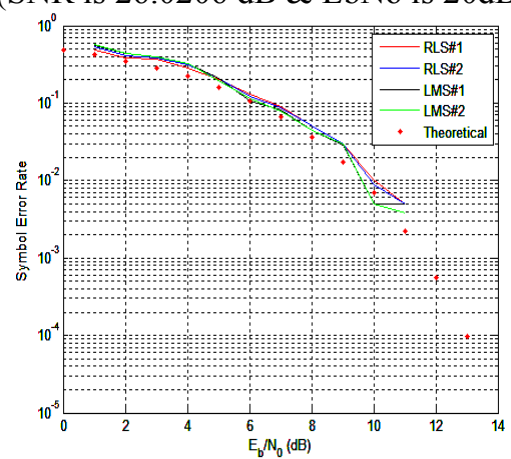

Figure 1.6: SER vs EbNo of the equalised signals using RLS and LMS algorithms at step size 0.0003 


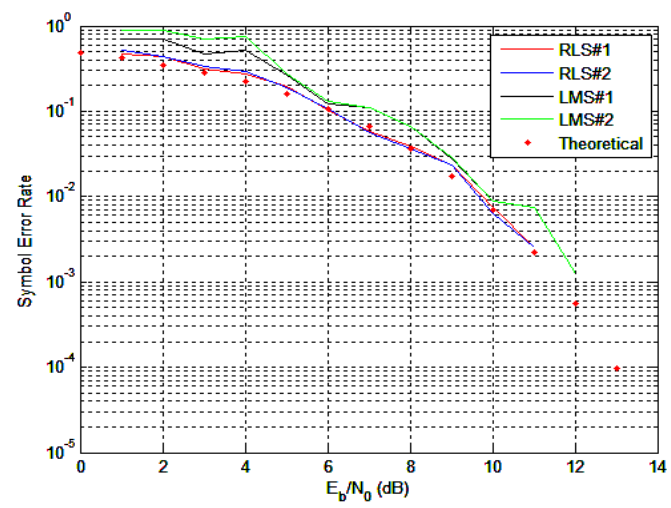

Figure 1.7: SER vs EbNo of the equalised signals using RLS and LMS algorithms at step size 0.003

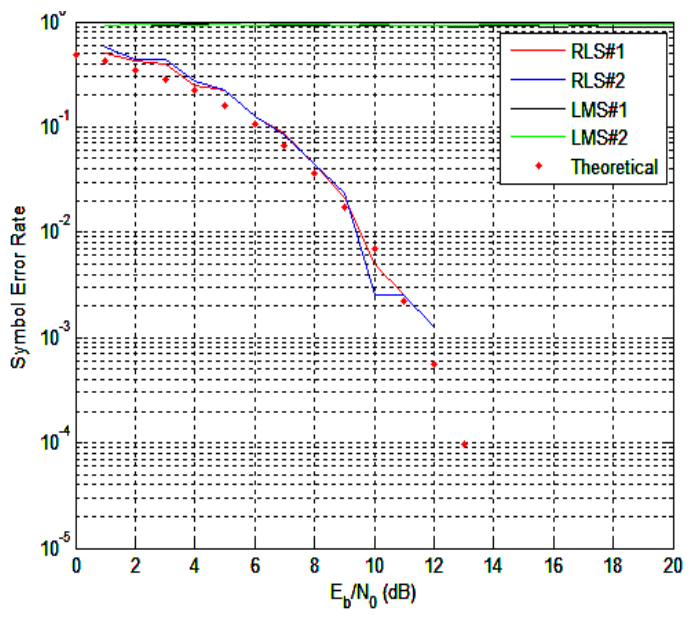

Figure 1.8: SER vs EbNo of the equalised signals using RLS and LMS algorithms at step size 0.03

From the results obtained, it is found that as the Step Size is decreased down towards zero, SER gets reduced and LMS and RLS tracks or match closely the theoretical predictions. When the Step Size increased towards 1, SER gets increased and Step - Size dependent LMS algorithm does not converge resulting a poor choice for equalization.

Thus, it is observed that Step Size is an important design criterion and plays a significant roll in the performance of LMS equalizer. It needs to be chosen appropriately to reach the desired criteria. Here we have found that the LMS equalizer is giving optimal performance at step size of 0.0003 .

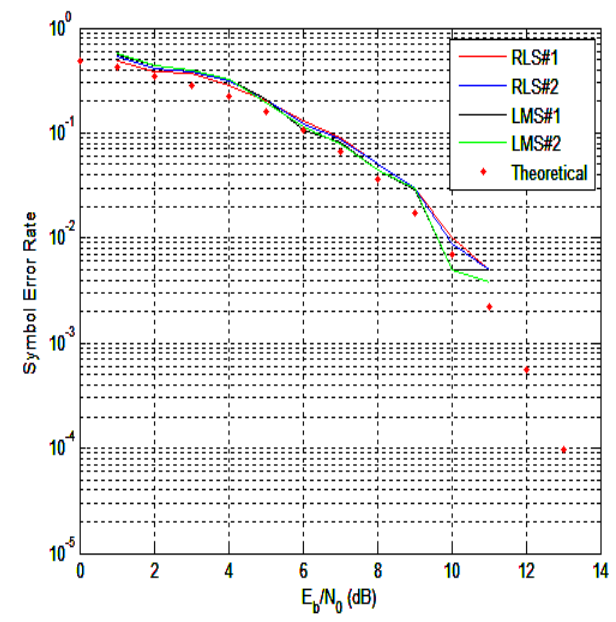

Figure 1.9: SER vs EbNo of the equalised signals using RLS and LMS algorithms with filter length 6 


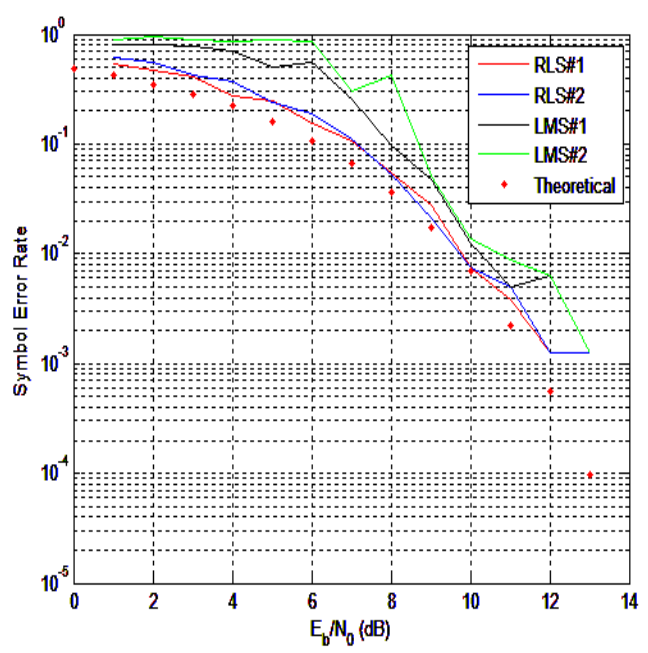

Figure 1.10: SER vs EbNo of the equalised signals using RLS and LMS algorithms with filter length 15

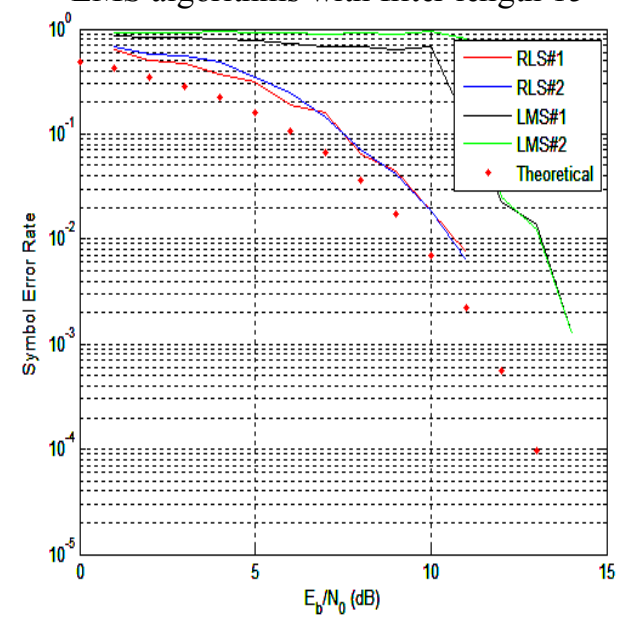

Figure 1.11: SER vs EbNo of the equalised signals using RLS and LMS algorithms with filter length 30

It is clearly demonstrated that with increase in no. of weights (filter length) for the Equalizer to be updated, the complexity of LMS and RLS algorithms to converge also increases. Initially the SER performance is investigated at 6 weights. In second case, the 15 no. of weights are taken and in third case weights to be updated are 30 .

It is observed that as the no. of weights increases, the Symbol Error Rate Increases. Thus no. of weights is also an important attribute or design criteria for the Equalizer.

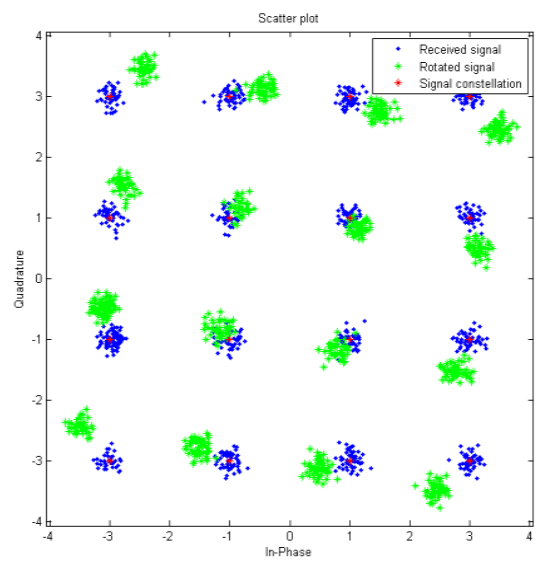

Figure 1.12: The received noisy modulated data with rotated phase of 10 degrees (SNR is $26.0206 \mathrm{~dB} \&$ EbNo is $20 \mathrm{~dB})$. 


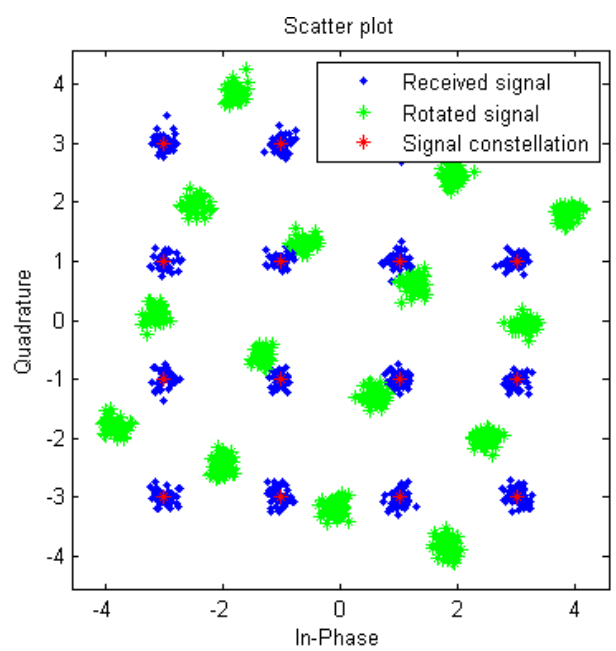

Figure 1.13: The received noisy modulated data with rotated phase of 20 degrees (SNR is $26 \mathrm{~dB} \&$ EbNo is 20dB).

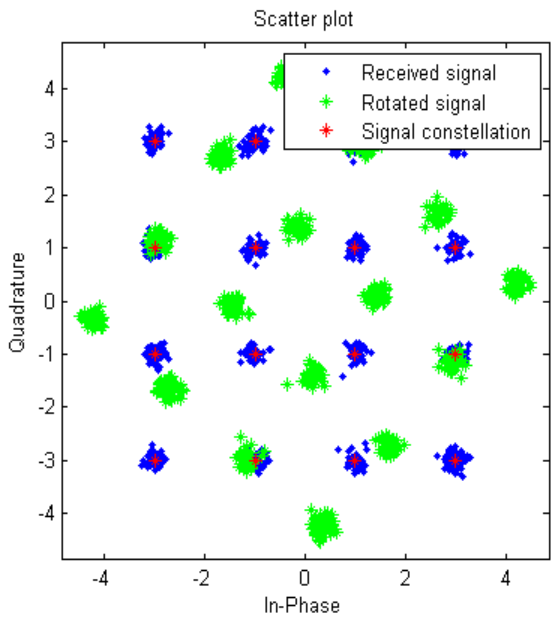

Figure 1.14: The received noisy modulated data with rotated phase of 40 degrees (SNR is $26 \mathrm{~dB} \&$ EbNo is $20 \mathrm{~dB})$

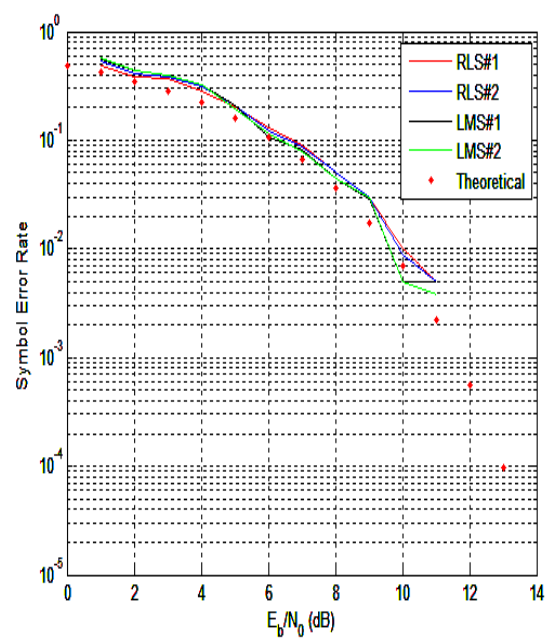

Figure 1.15: SER vs EbNo of the equalised signals using RLS and LMS algorithms with Phase Rotation 10 degree 


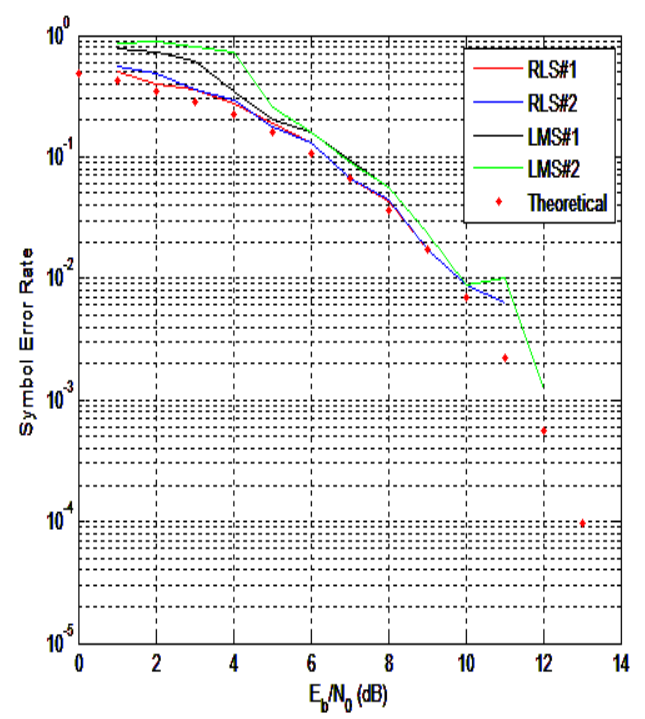

Figure 1.16: SER vs EbNo of the equalised signals using RLS and LMS algorithms with Phase Rotation 20 degree

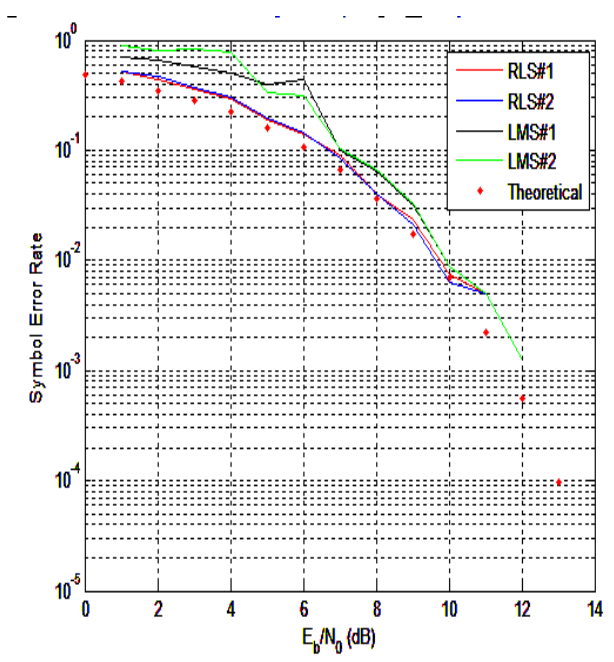

Figure 1.17: SER vs EbNo of the equalized signals using RLS and LMS algorithms with Phase Rotation 40 degree.

The same Observation is made in case when the phase offset of QAM modulated carrier is increased from 10 degrees to 40 degrees. It has also notices that RLS algorithm converges fastly than LMS algorithm. It is also observed that the SER is slightly higher for situation when the phase shift is high.

\section{Conclusion:}

It is observed that the SER is slightly higher for situations when the noise is high (EbNo is between 1 to 5). Overall, it can be concluded from the results that RLS converges fastly than LMS, but has comparatively higher complexity and No. of weights, Step Size, Phase Rotation, Forgetting Factor (Gain Factor) and the Constellation Size are the significant design criteria for both RLS equalizer and LMS equalizer and they need to be chosen appropriately to recover or estimate the phase of the carrier.

[1]. Simon Haykin," Adaptive Filter Theory", Prentice- hall, 2002

\section{Reference}

[2]. T. Arnantapunpong, T. Shimamura, and S. A. Jimaa," A New Variable Step Size for Normalized LMS Algorithm", NCSP'10 - 2010 RISP International Workshop on Nonlinear Circuits, Communications and Signal Processing, Honolulu, Hawaii, USA March 3-5, 2010 .

[3]. Y. Wang, C. Zhang, and Z. Wang, "A new variable step-size LMS Algorithm with application to active noise control”, Proc. IEEE ICASSP, pp. 573-575, 2003.

[4]. S. Hayking, A.H. Sayed, J.Zeidler, P.Yee, P.Wei, “Tracking of linear Time-Variant Systems,” Proc. MILCOM, pp.602-606, San Diego, Nov. 1995. 
[5]. H.Sadoghi Yazdi, M.Lotfizad, E.Kabir, M.Fathi "Applicattion of trajectory learning in tracking vehicles in the traffic scene" $9^{\text {th }}$ Iranian computer conference vol.1, pp. 180-187, Feb 2004.

[6]. O.P. Sharma, V. Janyani and S.Sancheti, "Recursive Least Squares Adaptive Filter a better ISI Compensator", International Journal of Electronics, Circuits and Systems, 2009.

[7]. R.H Kwong, E.W.Johnston, “A variable Step size LMS algorithm”, IEEE Trans Signal Processing, Vol.40,Issue.7,PP.1633-1642, July 1992

[8]. K.Banovic,Esam Abdel-Raheem, and Khalid, "Anovel Radius-Adjusted Approach for blind Adaptive Equalizer", IEEE Signal Processing Letters, Vol.13,No.1,pp.37 - 40, Jan. 2006.

[9]. S. Choi, T-Lee, D Hong." Adaptive error constrained method for LMS algorithm and application"Signal Processing . Vol. 85, Issue .10, PP. $1875-1897$, Oct. 2005.

[10]. Adinoyi, S. Al-Semari, A. Zerquine, "Decision feedback equalisation of coded I-Q QPSK in mobile radioenvironments," Electron. Lett. vol. 35, No1, pp. 13-14, Jan. 1999.

[11]. Wang Junfeng, Zhang Bo, "Design of Adaptive Equalizer Based on Variable Step LMS Algorithm," Proceedings of the Third International Symposium on Computer Science and Computational Technology (ISCSCT '10) Jiaozuo, P. R. China, 14-15, pp. 256258, August 2010.

[12]. Antoinette Beasley and Arlene Cole-Rhodes, "Performance of Adaptive Equalizer for QAM signals," IEEE,Military communications Conference, 2005. MILCOM 2005, Vol.4, pp. 2373 - 2377.

[13]. Mahmood Farhan Mosleh, Aseel Hameed AL-Nakkash, "Combination of LMS and RLS Adaptive Equalizer for Selective Fading Channel,” European Journal of Scientific Research ISSN 1450-216X Vol.43, No.1, pp.127-137, 2010.

[14]. Ehsan Hassani Sadi, Hamidreza Amindavar, "Blind Phase Recovery in QAM Communication systems using Characteristic Function", IEEE sponsored Conference ICASSP 2013 (978-1-4577-0539-7/13/ \$ 26.00 @ 2013 IEEE

[15]. M. Musso, M. gandetto, G. Gera, S. Canepa, R. Singh and C.S. regazzoni, A novel combined algorithms for $32-$ QAM carrier recovery, International Journal of Electronics and Communication Engineering, Volume 7, Number 3, ISSN 0974-2166, pp - 1-5, 2013

[16]. www. nptel.ac.in/introduction to adaptive equalizers

[17]. Digital Communication $4^{\text {th }}$ Edition by John G. Proakis 